\title{
A New Experimental Infection Model in Ferrets Based on Aerosolised Mycobacterium bovis
}

\section{Lyanne McCallan, ${ }^{1}$ David Corbett, ${ }^{1}$ Peter L. Andersen, ${ }^{2}$ Claus Aagaard, ${ }^{2}$ David McMurray, ${ }^{3}$ Claire Barry, ${ }^{1}$ Suzan Thompson, ${ }^{1}$ Samuel Strain, ${ }^{1}$ and Jim McNair ${ }^{1}$}

\author{
${ }^{1}$ Veterinary Sciences Division, Agri-food and Biosciences Institute, Stoney Road, Belfast BT4 3SD, UK \\ ${ }^{2}$ Department of Infectious Immunology, Statens Serum Institut, 5 Artillerivej, 2300 Copenhagen S, Denmark \\ ${ }^{3}$ Department of Microbial and Molecular Pathogenesis, Texas A\&M University System Health Science Center, College Station, \\ TX 77840-7896, USA
}

Correspondence should be addressed to Jim McNair, jim.mcnair@afbini.gov.uk

Received 13 January 2011; Revised 14 February 2011; Accepted 16 February 2011

Academic Editor: Mitchell V. Palmer

Copyright ( $\odot 2011$ Lyanne McCallan et al. This is an open access article distributed under the Creative Commons Attribution License, which permits unrestricted use, distribution, and reproduction in any medium, provided the original work is properly cited.

\begin{abstract}
There is significant interest in developing vaccines to control bovine tuberculosis, especially in wildlife species where this disease continues to persist in reservoir species such as the European Badger (Meles meles). However, gaining access to populations of badgers (protected under UK law) is problematic and not always possible. In this study, a new infection model has been developed in ferrets (Mustela furo), a species which is closely related to the badger. Groups of ferrets were infected using a Madison infection chamber and were examined postmortem for the presence of tuberculous lesions and to provide tissue samples for confirmation of Mycobacterium bovis by culture. An infectious dose was defined, that establishes infection within the lungs and associated lymph nodes with subsequent spread to the mesentery lymph nodes. This model, which emphasises respiratory tract infection, will be used to evaluate vaccines for the control of bovine tuberculosis in wildlife species.
\end{abstract}

\section{Introduction}

The persistence of the bovine tuberculosis problem in cattle and other species on a worldwide basis has led to the reappraisal of control programmes to counter this disease. The current direction for research into the control of bovine TB includes evaluation of vaccines that may be deployed in wildlife populations, including badgers (Meles meles) $[1,2]$. This work has been largely driven through an experimental model approach [3]. Despite considerable advances in our understanding of the pathogenesis and immunology of the disease and the impact of vaccination in a range of species, there remains a significant paucity of information relating to vaccine efficacy. Significant advances have been made through the use of M. bovis BCG; yet, some studies reported partial protection in badgers against experimental challenge $[3,4]$. There remains a need to evaluate other types of vaccines (protein subunit vaccines for example) that may induce enhanced protection from infection or even boost Mycobacterium bovis (M. bovis) BCG generated immunity [5].
The use of free ranging badgers taken from wildlife populations and held in captivity, to study vaccine efficacy, is problematic, primarily since the badger is a protected species under UK legislation and removal of wild animals for experimentation is very emotive to the public. Using a surrogate species, such as the ferret (Mustela furo), brings a number of advantages that include the availability of animals from licensed suppliers, the capacity to control a number of significant experimental features, and the opportunity to adopt a multifaceted approach to the study (controlled infection dose, pathogenesis, immunology, and culture confirmation).

The ferret has been used in various experimental models to study a number of human pathogens to which it is susceptible [6-8]. However, this species is also susceptible to infection with M. bovis [9] and it is closely related to the European badger (both are members of the Mustelidae), making it an attractive surrogate species in which to develop an aerosol infection model when wild badgers are unavailable. 
The main purpose of this study was to establish an experimental model of infection in the ferret using aerosolised $M$. bovis by defining the optimum challenge dose that could be used to induce disease. In doing so, we intend to use this model in future studies to measure the efficacy of protein subunit vaccines.

\section{Materials and Methods}

2.1. Experimental Rationale and Design. The purpose of this study was to establish an M. bovis infectious dose delivered by aerosol, to initiate infection and establish the disease in ferrets. This is part of a larger study to measure the efficacy of antibovine tuberculosis vaccines for wildlife species. In order to define the optimal $M$. bovis challenge dose, we infected ferret groups with either $1 \times 10^{2} \mathrm{CFU}$ or $5 \times 10^{3} \mathrm{CFU}$ of $M$. bovis strain AF2122/97 (a kind gift from Dr M. Vordermeier, VLA, Weybridge) and carried out postmortem examinations at 8 and 20 weeks post infection (PI).

Prior to the commencement of any procedures, approval to carry out ferret-based experiments was given by the UK Home Office and the AFBI Ethics Review Committee and all procedures were carried out in accordance with the United Kingdom Animals (Scientific Procedures) Act 1986.

2.2. Ferrets. Ferrets used in this experiment were born into litters within a two-week period and were fully weaned when they arrived into AFBI. Sixteen male ferrets were obtained from approved UK suppliers, through Harlan Laboratories, UK. Upon arrival at the biosecure containment level 3 suite, ferrets were split into two groups of 8 depending on litter and age and housed in bespoke pens with environmental enrichment. The mean age (SD) for the group of ferrets on arrival at AFBI was 6.4 (SD 0.51) weeks. At the time of infection, the mean age (SD) was $20.4(0.51)$ weeks. This building was maintained under negative air pressure, with high-efficiency particulate air (HEPA) filtration. In addition to cleaning each pen and replacing food (James Wellbeloved, UK) and water on a daily basis, each ferret was handled and examined for any signs of infection (demeanour, movement, bite wounds, etc.). The individual weight of each ferret was recorded on a weekly basis throughout the duration of the experiment to provide an additional measure of health.

2.3. Preparation of M. bovis for Aerosol Experiments. $M$. bovis strain AF2122/97, a virulent field strain isolated from a cow found to have caseous lesions in the lung and bronchomediastinal lymph nodes, was used as the inoculum for aerosol exposure. Unicellular M. bovis stock cultures were prepared as described previously [10]. For aerosol exposure, aliquots of unicellular M. bovis stock cultures were thawed and diluted to the desired concentration in sterile $0.01 \mathrm{M}$ phosphate buffered saline (PBS), pH 7.2 immediately before use.

2.4. Aerosol Exposure. All ferrets were exposed to M. bovis using a Madison aerosol chamber, essentially as described by Rodgers et al. [10]. Two groups of ferrets were established and were infected with either $1 \times 10^{2}$ or $5 \times 10^{3} \mathrm{CFU}$ M. bovis. Prior to infection unicellular cultures at $10^{6} \mathrm{CFU}$ per $\mathrm{mL}$, was placed in the aerosol chamber nebulizer jar with the exposure cycle pre set to deliver the appropriate infection dose. Following the timed exposure period, the infection chamber was purged with clean, filtered air and the ferret groups were returned to their pens. During each infection cycle the air stream carrying aerosolized $M$. bovis was sampled into an all glass impinger (AGI) containing phosphate buffered saline (PBS) to calculate the actual infective dose (CFU) delivered to each group. One hundred $\mu \mathrm{l}$ of PBS from each impinger (undiluted, 1 in 10, 1 in 100 and 1 in 1000 dilutions in PBS) were inoculated in quadruplicate onto 7H11-OADC agar plates and incubated at $37^{\circ} \mathrm{C}$ for up to 6 weeks.

2.5. Faecal Sampling. A sample of faeces was collected from the latrine area of each pen every week for the duration of the experiment, to monitor excretion of M. bovis in faeces. Samples were split into two aliquots and decontaminated using either $0.075 \%$ (final volume) hexadecylpyridinium chloride (HPC) or $5 \%$ (final volume) oxalic acid followed by centrifugation at $3000 \mathrm{~g}$ for $15 \mathrm{~min}$. Pellets were resuspended in $1 \mathrm{~mL} 0.01 \mathrm{M}$ sterile phosphate buffered saline ( $\mathrm{pH} 7.2$ ) and inoculated onto the BACTEC MGIT 960 culture system. Suspect $M$. bovis culture positive samples were stained using the Ziehl-Neelsen method. Samples from faeces shown to contain typical acid-fast rods were confirmed as M. bovis positive using Variable Number Tandem Repeat (VNTR) typing to confirm re-isolation of AF2122/97 [11].

2.6. Postmortem Examination and Lesion Score. During the design of this experiment, we decided to use the culture of $M$. bovis from tissues as the primary indicator for infection. The reason for this was that there was likely to be very limited amounts of tissue available for laboratory confirmation techniques. Any attempt to prepare tissues for bacteriology and histopathology would compromise both techniques and experience gained from previous experiments in cattle that demonstrated a superior sensitivity with culture techniques compared to histopathology. In addition, M. bovis induced lesions are difficult to discern in ferrets, with bacteriology greatly enhancing detection of $M$. bovis infection [12].

At the end of each experimental period, each ferret was euthanized using isofluorothane gas followed by pentobarbitone overdose and examined for the presence of tuberculous lesions. Prior to dissection, the fur on each animal was clipped and washed in chlorhexidine surgical scrub to reduce the potential for microbial contamination. Carcasses were dissected and tissue samples carefully removed into sterile containers for further examination and for preparation prior to inoculation onto culture media. During carcass dissection, a standardised protocol was followed to ensure consistency and accuracy. Firstly, the lungs were removed and cut into four quarters representing right upper and lower and left upper and lower sections. The kidneys, liver, and spleen were then removed followed by the mesenteric lymph node. Finally, the submandibular lymph nodes were removed and, if visible, the retropharyngeal lymph nodes. Prior to 
preparation for culture, all tissues were dissected in detail to identify and measure lesions, if present.

Tuberculous lesions, if present, were measured, recorded and assigned a lesion score according to their presence and extent. When lesions were absent, a score of zero was recorded. If one small focus of infection was seen a score of 1 was recorded. When several foci or an area of necrosis (measuring $5 \mathrm{~mm}$ or greater) was observed, a score of 2 was given and when multiple lesions and/or extensive necrosis was observed, a score of 3 was recorded. Only lesions that had been confirmed as tuberculosis following isolation of M. bovis were used in the analysis of postmortem scores. All tissues removed for examination and culture were assigned a postmortem score.

All postmortem examinations were carried out within the containment level three suite, under negative air pressure, where complete sterility could not be guaranteed. For this reason, all tissue samples were considered to be potentially contaminated through exposure to the environment.

2.7. Preparation of Tissue Homogenates for Culture. A total of 156 samples were submitted for both qualitative and quantitative bacteriological culture at 8 weeks and 20 weeks PI, with sampled tissues including the submandibular, mesenteric, and lung-associated lymph nodes (LNs), as well as the lungs, liver, spleen, and kidneys. Each tissue sample was trimmed to remove fat, weighed, dissected carefully to reveal lesions indicative of tuberculosis and then sliced into approximately $5 \mathrm{~mm}$ cubes. The cubed tissue samples were then placed into double thickness stomacher bags (Seward, Worthington, UK) with between 5 and $10 \mathrm{~mL}$ PBS (depending on the weight of sample) and homogenised in a stomacher (Biomaster 80, Seward) at high speed for $2 \mathrm{~min}$. Tissue homogenates were decanted into sterile universal bottles for inoculation into media for qualitative and quantitative culture. Urine was recovered from the bladder in most cases but not in sufficient volumes to allow a full bacteriological analysis. Despite this, samples were swabbed onto 7H11-OADC agar plates and incubated at $37^{\circ} \mathrm{C}$ for up to 6 weeks.

2.8. Qualitative Culture. For qualitative culture, tissue homogenates were inoculated into the BACTEC MGIT 960 culture system and incubated for up to 56 days. Samples identified as positive by the BACTEC MGIT 960 system were stained using the Ziehl-Neelsen method. Samples containing typical acid-fast rods were presumed to be positive for $M$. bovis and analysed further by VNTR to indentify the strain [11].

2.9. Quantitative Culture. For quantitative culture, $100 \mu \mathrm{l}$ of tissue homogenates (undiluted, 1 in 10, 1 in 100 and 1 in 1000 dilutions in PBS) were inoculated in quadruplicate onto $7 \mathrm{H} 11-\mathrm{OADC}$ agar plates and incubated at $37^{\circ} \mathrm{C}$ for up to 6 weeks. Tissue homogenates were decontaminated in $0.075 \%$ (final volume) hexadecylpyridinium chloride (HPC) prior to inoculation onto 7H11-OADC agar plates. M. bovis colonies were counted weekly from 3 weeks onwards and the average colony counts at 6 weeks post-inoculation used to calculate the total number of CFU per $\mathrm{g}$ of sample. M. bovis colonies were initially identified on the basis of colony morphology and a selection of colonies were prepared for VNTR typing to confirm isolation of AF2122/97 [11].

2.10. Data Analysis. Where appropriate, data was analysed using GraphPad InStat version 3.05 for Windows NT. Data was analysed by one-way analysis of Variance (ANOVA) with Tukey-Kramer multiple comparisons test.

\section{Results}

3.1. Clinical Observations. All ferrets were examined on a daily basis for signs of ill health and bite wounds, were observed feeding and drinking regularly, and appeared to be in good health during the entire experimental period. During this period, no adverse clinical signs were observed in any of the ferrets. A number of ferrets had bite wounds between the shoulders, but these were considered to be minor and did not warrant treatment. The weight of each ferret was recorded every week and used as an indirect measure of health status. During the experimental period, all ferrets either maintained or gained weight, with the exception of two animals where the weight loss was $4.8 \%$ and $12.8 \%$. There was no significant difference between the mean weight of each group at time point zero (preinfection) or at the time when postmortem examinations were carried out (data not shown).

3.2. Aerosol Exposure. At the outset, the intention was to infect each group of ferrets with either $1 \times 10^{2} \mathrm{CFU}$ or $5 \times 10^{3}$ CFU M. bovis. The actual dose delivered was estimated by capturing aerosolised $M$. bovis from the airstream delivered to the Madison chamber for culture onto 7H11 media. The actual dose delivered to each group was calculated to be $0.8 \times 10^{2} \mathrm{CFU}$ (lower dose) and $1.1 \times 10^{3} \mathrm{CFU}$ (higher dose).

3.3. Culture of $M$. bovis from Faeces. A total of 49 faeces samples were collected during the experiment for qualitative culture using the BACTEC MGIT 960 system. Samples collected prior to infection were culture negative for $M$. bovis. After infection and up to 20 weeks after infection (end of experiment), a total of 10 faeces samples were found to be positive for M. bovis (between 5 and 18 weeks post infection), of which nine originated from the group infected with $1.1 \times 10^{3} \mathrm{CFU}$. Isolates obtained from all ten positive faeces were confirmed as AF2122/97 using the VNTR typing method. Culture positive faeces samples were only detected by the BACTEC MGIT 960 system after decontamination with $5 \%$ oxalic acid. Corresponding samples decontaminated with $0.075 \%$ HPC were negative at the end of the incubation period (56 days).

3.4. Postmortem Examination and Lesion Score. Ferrets were euthanised at either 8 or 20 weeks PI and examined postmortem. The greater number of lesions confirmed as tuberculous, was found in the lungs and lung-associated lymph nodes. When present, suspect lesions had the appearance of discrete, pale, circumscribed areas which were not 
TABLE 1: Isolation of $M$. bovis from a range of tissues taken from 4 ferrets infected with $0.8 \times 10^{2} \mathrm{CFU}$ and examined postmortem at 8 weeks post infection.

\begin{tabular}{|c|c|c|c|c|c|c|c|c|c|c|c|c|}
\hline \multirow{2}{*}{$\begin{array}{l}\text { Tag number } \\
\text { Tissue }\end{array}$} & \multicolumn{3}{|c|}{432} & \multicolumn{3}{|c|}{481} & \multicolumn{3}{|c|}{523} & \multicolumn{3}{|c|}{564} \\
\hline & $\mathrm{LS}^{1}$ & $\mathrm{QL}^{2}$ & $\mathrm{QN}^{3}$ & LS & QL & QN & LS & QL & QN & LS & QL & QN \\
\hline Submandibular LN & 0 & - & - & 0 & - & - & 0 & - & - & 0 & + & 24.3 \\
\hline Retropharyngeal LN & 0 & - & - & $\mathrm{NS}^{4}$ & NS & NS & NS & NS & NS & 0 & + & 31.7 \\
\hline Mesenteric LN & 0 & + & 1.94 & 0 & + & 16.3 & 0 & - & 32.8 & 1 & + & 1080 \\
\hline Upper left lung & 0 & - & - & 0 & + & 2.96 & 0 & - & - & 0 & + & 36.8 \\
\hline Lower left lung & 0 & + & - & 0 & + & - & 0 & - & 74.4 & 0 & + & 14.9 \\
\hline Upper right lung & 0 & + & 5.4 & 0 & + & - & 0 & - & - & 0 & + & - \\
\hline Lower right lung & 0 & + & - & 0 & + & 32 & 0 & - & 81.8 & 0 & + & - \\
\hline Lung associated LN & 0 & + & - & 0 & + & 11.8 & 2 & - & 1190 & 1 & + & 46.6 \\
\hline Kidney & 0 & - & - & 0 & - & - & 0 & - & - & 0 & 0 & 152 \\
\hline Liver & 0 & - & 88 & 0 & + & - & 0 & - & - & 0 & + & - \\
\hline Spleen & 0 & + & - & 0 & - & - & 0 & - & - & 0 & + & 8.5 \\
\hline Kidney (2) & 0 & - & - & NS & NS & NS & NS & NS & NS & NS & NS & NS \\
\hline
\end{tabular}

${ }^{1}$ LS: lesion score.

${ }^{2} \mathrm{QL}$ : qualitative bacteriology.

${ }^{3} \mathrm{QN}$ : quantitative bacteriology $\left(\times 10^{2} \mathrm{CFU} / \mathrm{g}\right)$.

${ }^{4}$ NS: not sampled.

necrotic or caseous. Typically, lesions were less than $5 \mathrm{~mm}$ in diameter and did not coalesce. In each infection group, the tissues where lesions were most likely disclosed were the lungs and lung associated lymph nodes. Postmortem scores ranged between 1 and 3 and were consistently higher with the greater infection dose (Tables 1 to 4 ). After the lungs, the mesenteric lymph nodes were the site most likely to find lesions, although the postmortem scores were lower, between 1 and 2. Only three additional lesions were found with a PM score between 1 and 2, and these were in the spleen tissue of ferrets infected with $1 \times 10^{3} \mathrm{CFU}$ (Tables 1-4). A range of tissues were examined and sampled for bacterial culture. These included the lungs, lung-associated lymph nodes, the submandibular and mesenteric lymph nodes, spleen, liver, and kidneys. If visible, the retropharyngeal lymph nodes were also removed. When examined at 8 weeks PI, the mean postmortem score for lesions disclosed in the group infected with $0.8 \times 10^{2} \mathrm{CFU}$ was 1 (SEM 0.56) compared to 9.25 (SEM 1.1) in the group infected with $1.1 \times 10^{3}$ CFU. A mean postmortem score of 1.25 (SEM 1.25) was calculated for the group infected with $0.8 \times 10^{2} \mathrm{CFU}$ and examined postmortem at 20 weeks, compared to 7.75 (SEM 1.31) for the group infected with $1.1 \times 10^{3} \mathrm{CFU}$ examined at 20 weeks PI (Figure 1). The differences in the postmortem score between groups infected with $0.8 \times 10^{2}$ and $1.1 \times 10^{3} \mathrm{CFU}$ and examined at 8 weeks PI were statistically significant $(P<.001)$. Differences were also statistically significant at the $P<.01$ level between the group infected with $0.8 \times$ $10^{2}$, examined at 8 weeks PI and the group infected with $1.1 \times 10^{3} \mathrm{CFU}$ at 20 weeks PI, and the group infected with $0.8 \times 10^{2} \mathrm{CFU}$ at 20 weeks PI compared to the group infected with $1.1 \times 10^{3} \mathrm{CFU}$ and examined at 8 weeks PI (Figure 1).
3.5. Culture of M. bovis from Tissue Samples. During postmortem examination, a number of urine samples were removed for culture; however, $M$. bovis was not isolated from any of these samples. Of the 156 tissue samples submitted, $M$. bovis was isolated from 102 samples (65\%) using a combination of qualitative and quantitative culture. Isolates obtained from a representative panel of culture positive samples were confirmed as AF2122/97, by VNTR. The number of samples from which $M$. bovis was isolated (qualitative culture), and the concentration of M. bovis $\left(\mathrm{CFU} / \mathrm{g} \times 10^{2}\right)$ in the samples (quantitative culture) is shown in Tables 1 to 4 .

At 8 weeks PI, M. bovis was isolated from 29/43 samples (69\%) from the group infected with $0.8 \times 10^{2} \mathrm{CFU}$ using a combination of qualitative and/or quantitative culture compared to $30 / 42$ samples $(71 \%)$ from the group infected with $1.1 \times 10^{3} \mathrm{CFU}$. Estimates of bacterial counts varied widely within both groups examined at 8 weeks PI (Tables 1 and 2). The differences between mean group values for bacterial load were not statistically significant. In both the $0.8 \times 10^{2}$ and $1.1 \times 10^{3} \mathrm{CFU}$ groups at 8 weeks PI, M. bovis was consistently isolated from the lower respiratory tract (lungs, lung-associated lymph nodes) and mesenteric lymph nodes with only occasional isolation from the extrapulmonary sites (submandibular and retropharyngeal LN, kidney, liver, and spleen).

At 20 weeks PI, M. bovis was isolated from 16/40 (40\%) samples from the group infected with $0.8 \times 10^{2} \mathrm{CFU}$ using a combination of qualitative and/or quantitative culture compared to $32 / 40(80 \%)$ samples from the group infected with $1.1 \times 10^{3} \mathrm{CFU}$. Estimates of bacterial counts varied widely within both groups examined at 20 weeks PI (Tables 3 and 4). The differences between mean group values for 
TABLE 2: Isolation of $M$. bovis from a range of tissues taken from 4 ferrets infected with $1.1 \times 10^{3} \mathrm{CFU}$ and examined postmortem at 8 weeks post infection.

\begin{tabular}{|c|c|c|c|c|c|c|c|c|c|c|c|c|}
\hline \multirow{2}{*}{$\begin{array}{l}\text { Tag number } \\
\text { Tissue }\end{array}$} & \multicolumn{3}{|c|}{349} & \multicolumn{3}{|c|}{358} & \multicolumn{3}{|c|}{728} & \multicolumn{3}{|c|}{884} \\
\hline & $\mathrm{LS}^{1}$ & $\mathrm{QL}^{2}$ & $\mathrm{QN}^{3}$ & LS & QL & QN & LS & QL & QN & LS & QL & QN \\
\hline Submandibular LN & 0 & + & - & 0 & - & - & 0 & - & - & 0 & - & - \\
\hline Retropharyngeal LN & $\mathrm{NS}^{4}$ & NS & NS & 0 & - & - & NS & NS & NS & 0 & - & - \\
\hline Mesenteric LN & 1 & + & - & 2 & + & - & 1 & + & - & 1 & + & - \\
\hline Upper left lung & 1 & + & - & 0 & + & - & 2 & + & - & 2 & + & - \\
\hline Lower left lung & 2 & + & 34 & 1 & + & - & 2 & + & - & 0 & - & - \\
\hline Upper right lung & 2 & + & 21.6 & 0 & + & - & 2 & + & - & 0 & - & - \\
\hline Lower right lung & 2 & + & 21.7 & 2 & + & - & 3 & + & - & 2 & + & - \\
\hline Lung associated LN & 0 & + & 52.3 & 2 & + & - & 2 & + & 7 & 2 & + & - \\
\hline Kidney & 0 & - & - & 0 & - & - & 0 & - & 0.22 & 0 & - & - \\
\hline Liver & 0 & - & 38.5 & 0 & - & - & 0 & + & 26.3 & 0 & - & - \\
\hline Spleen & 2 & + & 51.1 & 1 & + & - & 0 & + & 0.19 & 0 & - & 2.31 \\
\hline
\end{tabular}

${ }^{1}$ LS: lesion score.

${ }^{2} \mathrm{QL}$ : qualitative bacteriology.

${ }^{3} \mathrm{QN}$ : quantitative bacteriology $\left(\times 10^{2} \mathrm{CFU} / \mathrm{g}\right)$.

${ }^{4}$ NS: not sampled.

TABLE 3: Isolation of $M$. bovis from a range of tissues taken from 4 ferrets infected with $0.8 \times 10^{2}$ CFU and examined postmortem at 20 weeks post infection.

\begin{tabular}{|c|c|c|c|c|c|c|c|c|c|c|c|c|}
\hline \multirow{2}{*}{$\begin{array}{l}\text { Tag number } \\
\text { Tissue }\end{array}$} & \multicolumn{3}{|c|}{262} & \multicolumn{3}{|c|}{456} & \multicolumn{3}{|c|}{940} & \multicolumn{3}{|c|}{78968} \\
\hline & $\mathrm{LS}^{1}$ & $\mathrm{QL}^{2}$ & $\mathrm{QN}^{3}$ & LS & QL & QN & LS & QL & QN & LS & QL & QN \\
\hline Submandibular LN & 0 & - & - & 0 & - & 2.1 & 0 & - & - & 0 & - & - \\
\hline Retropharyngeal LN & $\mathrm{NS}^{4}$ & NS & NS & NS & NS & NS & NS & NS & NS & NS & NS & NS \\
\hline Mesenteric LN & 1 & + & 25.7 & 0 & + & & 0 & + & 70 & 0 & + & 7.0 \\
\hline Upper left lung & 2 & + & - & 0 & - & 417 & 0 & - & - & 0 & - & - \\
\hline Lower left lung & 2 & + & - & 0 & - & - & 0 & - & - & 0 & - & - \\
\hline Upper right lung & 0 & - & - & 0 & - & - & 0 & - & - & 0 & - & - \\
\hline Lower right lung & 0 & - & - & 0 & - & - & 0 & - & - & 0 & - & - \\
\hline Lung associated LN & 0 & + & 62.5 & 0 & + & - & 0 & - & - & 0 & + & 80.6 \\
\hline Kidney & 0 & - & - & 0 & - & - & 0 & - & - & 0 & - & - \\
\hline Liver & 0 & + & - & 0 & + & - & 0 & - & - & 0 & - & - \\
\hline Spleen & 0 & + & 0.78 & 0 & + & - & 0 & - & - & 0 & + & - \\
\hline
\end{tabular}

${ }^{1}$ LS: lesion score.

${ }^{2} \mathrm{QL}$ : qualitative bacteriology.

${ }^{3} \mathrm{QN}$ : quantitative bacteriology $\left(\times 10^{2} \mathrm{CFU} / \mathrm{g}\right)$.

${ }^{4} \mathrm{NS}$ : not sampled.

bacterial load were not statistically significant. At 20 weeks PI, M. bovis was isolated from all of the mesenteric and lungassociated LN cultured from the $0.8 \times 10^{2}$ CFU infection group, whereas $M$. bovis was isolated on only a few occasions from lungs, and extra-pulmonary sites. In contrast, $M$. bovis was isolated consistently from the lungs and spleen as well as the lung associated $\mathrm{LN}$ and mesenteric $\mathrm{LN}$ in the $1.1 \times 10^{3}$ CFU group at 20 weeks, with only occasional isolation from kidney and liver tissues. M. bovis was isolated from only one submandibular LN in the $0.8 \times 10^{2} \mathrm{CFU}$ infection group at 20 weeks PI.

3.6. Strain Confirmation by VNTR. In total, 65 putative $M$. bovis isolates from AGI, faeces, and tissue samples were analysed further by VNTR. All were confirmed to be M. bovis strain AF2122/97. 
TABLE 4: Isolation of M. bovis from a range of tissues taken from 4 ferrets infected with $1.1 \times 10^{3} \mathrm{CFU}$ and examined postmortem at 20 weeks post infection.

\begin{tabular}{|c|c|c|c|c|c|c|c|c|c|c|c|c|}
\hline \multirow{2}{*}{$\begin{array}{l}\text { Tag number } \\
\text { Tissue }\end{array}$} & \multicolumn{3}{|c|}{015} & \multicolumn{3}{|c|}{507} & \multicolumn{3}{|c|}{780} & \multicolumn{3}{|c|}{89968} \\
\hline & $\mathrm{LS}^{1}$ & $\mathrm{QL}^{2}$ & $\mathrm{QN}^{3}$ & LS & QL & QN & LS & $\mathrm{QL}$ & $\mathrm{QN}$ & LS & QL & QN \\
\hline Submandibular LN & 0 & - & - & 0 & - & - & 0 & - & - & 0 & - & - \\
\hline Retropharyngeal LN & NS & NS & NS & NS & NS & NS & NS & NS & NS & NS & NS & NS \\
\hline Mesenteric LN & 1 & + & 239 & 0 & + & 20.6 & 0 & + & 224 & 2 & + & 11.4 \\
\hline Upper left lung & 0 & + & 2.1 & 3 & + & 387 & 3 & + & 0.21 & 0 & + & - \\
\hline Lower left lung & 0 & + & 2.98 & 1 & + & 13.6 & 1 & + & 6.7 & 1 & + & - \\
\hline Upper right lung & 0 & + & 0.45 & 3 & + & 1.5 & 3 & + & 2.45 & 0 & - & - \\
\hline Lower right lung & 2 & + & 0.39 & 3 & + & 2.3 & 3 & + & 4.82 & 2 & + & - \\
\hline Lung associated LN & 1 & + & 1550 & 0 & + & 4.3 & 0 & + & 3490 & 0 & + & 9.38 \\
\hline Kidney & 0 & - & - & 0 & - & 19.1 & 0 & + & - & 0 & - & - \\
\hline Liver & 1 & - & 0.63 & 0 & - & - & 0 & + & 9.48 & 0 & + & - \\
\hline Spleen & 0 & + & 0.81 & 0 & + & - & 0 & + & 25.5 & 1 & + & - \\
\hline
\end{tabular}

${ }^{1}$ LS: lesion score.

${ }^{2} \mathrm{QL}$ : qualitative bacteriology.

${ }^{3} \mathrm{QN}$ : quantitative bacteriology $\left(\times 10^{2} \mathrm{CFU} / \mathrm{g}\right)$.

${ }^{4}$ NS: not sampled.

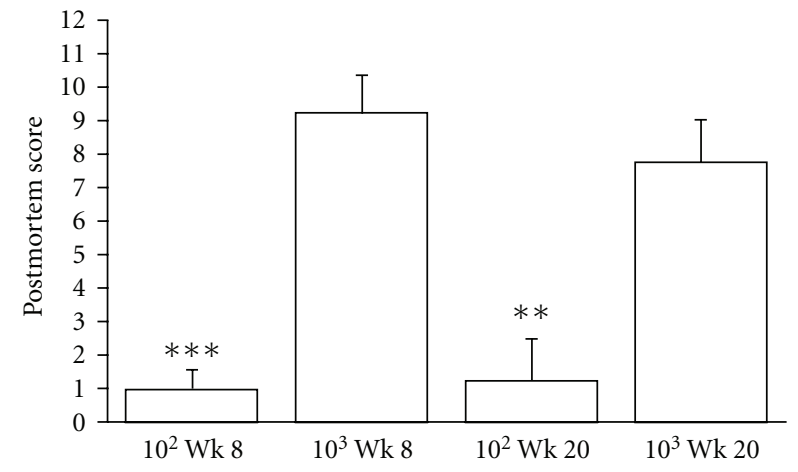

FIGURE 1: The mean postmortem scores for each group of ferrets infected with either $0.8 \times 10^{2}$ or $1.1 \times 10^{3}$ CFU M. bovis and examined postmortem at either 8 or 20 weeks post infection. Differences between the $10^{2}$ and $10^{3} \mathrm{CFU}$ infection groups at 8 weeks post infection were significant at the $P<.001$ level, while differences at week 20 post infection were significant at the $P<.01$ level.

\section{Discussion}

The purpose of this study was to establish an experimental model of infection in the ferret, using $M$. bovis culture as the main read out, that would induce disease and prove suitable for the future evaluation of vaccine efficacy. In addition, the lower respiratory tract was targeted in this experiment since bovine tuberculosis is principally an infectious disease of the respiratory system $[13,14]$. Some tissues from the upper respiratory tract (submandibular lymph nodes) were removed for culture while others (retropharyngeal lymph nodes) proved very difficult to locate. No attempts were made to sample nasal mucus, for example, due to the need for frequent anaesthesia and its inherent risks. This rationale follows the findings summarised by Corner et al. [15]. The immunology of vaccination and challenge in the ferret infection model will be described elsewhere.

At the outset, we intended to deliver two infection doses of $M$. bovis with a 50 -fold difference in CFU, in order to measure the impact of dose on the establishment of infection. However, the actual difference between the two doses was 14 -fold but we were still able to observe differences between these groups at 8 and 20 weeks post infection. At postmortem examination a number of tissues appeared to be tuberculous and only those lesions where infection was subsequently confirmed by culture, were assigned a postmortem score and used in analysis. Differences in lesion score between the two infection groups were statistically significant $(P<.001)$. It would appear that despite administration of a heavier infection dose and a longer infection period, there was not a greater disease burden at 20 weeks post infection. This observation could be explained by a lack of biological significance between the two infection doses. However, since there was no significant development of disease as measured by culture confirmed lesions, this suggests that ferrets, as with badgers, may become latently infected [14, 15], rather than progress toward a more severe form of the disease.

Bacteriological culture provided a reliable and specific method for the isolation of the infection strain (M. bovis AF2122/97) from tissue samples obtained in this experiment. The combination of qualitative (BACTEC MGIT) and quantitative (7H11-OADC agar plates) culture resulted in isolation of $M$. bovis from $67 \%$ tissue samples submitted for culture, including decontaminated and recultured samples. In total, $69 \%$ M. bovis positive samples were detected by both qualitative and quantitative culture. $M$. bovis was isolated from a further $41 \%$ samples by qualitative culture alone and $11 \%$ samples by quantitative culture alone. These results illustrate that neither culture method, when used 
individually, was capable of detecting all of the M. bovis positive samples and emphasises the importance of applying more than one culture method to maximise recovery of viable mycobacteria [16-19].

Bacterial counts varied widely with the lowest count observed in the $0.8 \times 10^{2}$ group examined at 8 weeks PI while the highest bacterial count was observed in the $1.1 \times$ $10^{3}$ group examined at 20 weeks. Tissues which consistently had the highest bacterial counts included the lungs, lungassociated lymph node, and mesenteric lymph nodes, with lower bacterial counts commonly observed in tissues such as the liver, spleen, and kidney. This is a feature of the aerosol infection method [10] and confirms delivery of M. bovis to the lower respiratory tract tissues of the lung $[10,20,21]$ where infection was established. However, the finding that all mesenteric lymph nodes were infected was surprising and less easily explained. Irrespective of bacterial load, each ferret was found to have infected mesentery lymph nodes, a finding that could be peculiar to this model using this route of infection and infectious dose. Lugton et al. (1997) concluded that in naturally infected New Zealand ferrets, infection was acquired primarily by the oral route, most likely through scavenging TB-infected carrion [22]. In over $60 \%$ of naturally infected ferrets with gross lesions, the mesentery lymph nodes were found to be infected. These findings differ from those reported in this study where the route of infection and, likely, the infectious dose were very different. Gallagher et al. reported faecal excretion in $65 \%$ of naturally infected badgers with pulmonary lesions, with between $10^{2}$ and $10^{5} \mathrm{CFU} M$. bovis per gram of faeces [23]. Exposure initiated by inhalation of M. bovis can establish infection in the lungs with subsequent spread by haematogenous dissemination to distal lymph nodes, and visceral organs $[24,25]$. There were no significant differences in the mean bacterial counts between the groups. However, there was considerable range in the numbers of bacteria recovered. This confirms the pattern of distribution for $M$. bovis primarily to the lung with spread to the mesenteric LN. Spread of infection to the kidneys, spleen, and liver was not confined to those ferrets with high bacterial loads in the lungs. Although this study was based on small numbers of ferrets in each infection group, this data suggests that spread of infection between various organs was influenced by a factor or factors other than bacterial load. Currently, we do not have sufficient additional data (histopathology, e.g.) to explain these findings but analysis of immunological data, when available, may provide clarification.

Faecal samples were taken from the latrine area of each pen to determine if $M$. bovis was excreted during the infection period. It was important to establish whether or not faecal excretion was a feature of this model, and, if so, would vaccination experiments (as part of the long term strategy) prevent release from infected animals? A small proportion of faecal samples were positive by BACTEC MGIT culture, with a clear bias towards groups receiving $1.1 \times 10^{3} \mathrm{CFU}$. Faecal excretion is not surprising since M. bovis was isolated from the mesenteric LN of all ferrets. Interestingly, M. bovis was isolated from faecal samples treated with oxalic acid but not from corresponding samples treated with HPC, indicating greater toxicity of HPC for M. bovis cells. This is in sharp contrast to previous published findings relating to use of decontamination agents [26]; however, much of this work was carried out with tissue samples rather than faecal samples. The recovery of M. bovis from faeces indicates excretion of viable bacteria, which could potentially act as a source of reinfection for ferrets. It is difficult to determine whether reinfection occurred via the oral route in this experiment. In field cases of TB in ferrets gross lesions are often observed in LN draining the alimentary tract, particularly the retropharyngeal and mesenteric LN [9, 27, 28]. In addition, over $60 \%$ of ferrets displaying gross lesions at a single tissue site also had infection of the mesenteric LN [27]. Such observations have led to the assumption that TB in wild ferrets is transmitted via the oral route through scavenging of TB-infected carcasses [24], an infectious dose which is necessarily higher than doses given via the respiratory route [29].

However, Cross et al. described oral inoculation in ferrets where $M$. bovis was isolated from those lymph nodes draining the head and mesenteries, with spread to the thoracic area, possibly by bacteraemia [30]. In our model, this mechanism may also account for the spread from the thoracic cavity to the mesenteries. It is unlikely that coprophagy contributed to reinfection or enhanced spread, since typically, bacterial load was less in mesenteric lymph nodes compared to the lungs.

Despite the differences in the two intended doses, aerosol infection establishes infection primarily in the lungs and associated lymph nodes with spread to the mesentery lymph nodes and occasionally to the liver, spleen, and kidneys. The model described here resembles the natural infection seen in badgers where the infection is principally found in the respiratory tract [14]. It is also clear that isolation of M. bovis by culture is sensitive and specific; however, reliance on one culture method alone is not sufficient to maximise recovery of $M$. bovis from a range of tissues.

The development of an infection model in ferrets to measure vaccine efficacy is not novel. Qureshi et al. reported partial protection in ferrets vaccinated with $M$. bovis BCG and challenged with $M$. bovis, both delivered by the oral route [31]. The challenge dose for this study was $5 \times 10^{6}$ CFU M. bovis, considerably greater that the dose given to ferrets in the current study. However, proof of principle was established with demonstration of immunity to infection. In future studies, the authors aim to refine ferret vaccination and challenge to identify vaccines that are effective in the control of bovine tuberculosis.

\section{Conclusions}

From the findings described above, we have established an infection model in the ferret. There are a number of similarities between this model and the natural disease seen in badgers, including the establishment of respiratory disease, spread of infection to the viscera and in the potential to establish latent infection. These factors indicate that this model is pertinent to the study of bovine tuberculosis in badgers. Given the phylogenetic similarity of badgers and 
ferrets, there is a distinct advantage in developing this model further to study vaccines designed to control $M$. bovis infection in wildlife species. Some questions remain unanswered; however, analysis of the immunological data from this study may clarify the role of this model and contribute toward a clearer understanding of the immune responses that follow infection and vaccination.

\section{Acknowledgments}

The authors are indebted to the staff of the TB Immunology Section for practical help with maintenance and care of the ferrets and with assistance during preparations for culture techniques. This study is in part funded by TB STEP KBBE-2007-1-3-04: strategies for the eradication of bovine tuberculosis (212414). The authors thank Mr. Carl McCormick for technical excellence with VNTR.

\section{References}

[1] R. J. Delahay, G. J. Wilson, G. C. Smith, and C. L. Cheeseman, "Vaccinating badgers (Meles meles) against Mycobacterium bovis: the ecological considerations," The Veterinary Journal, vol. 166, no. 1, pp. 43-51, 2003.

[2] M. A. Chambers, F. Rodgers, R. J. Delahay et al., "Bacillus Calmette-Guerin vaccination reduces the severity and progression of tuberculosis in badgers," Proceedings of the Royal Society B. In Press.

[3] L. A. L. Corner, E. Costello, S. Lesellier, D. O'Meara, and E. Gormley, "Vaccination of European badgers (Meles meles) with BCG by the subcutaneous and mucosal routes induces protective immunity against endobronchial challenge with Mycobacterium bovis," Tuberculosis, vol. 88, no. 6, pp. 601-609, 2008.

[4] S. Lesellier, L. Corner, E. Costello et al., "Antigen specific immunological responses of badgers (Meles meles) experimentally infected with Mycobacterium bovis," Veterinary Immunology and Immunopathology, vol. 122, no. 1-2, pp. 35-45, 2008.

[5] J. Dietrich, K. Weldingh, and P. Andersen, "Prospects for a novel vaccine against tuberculosis," Veterinary Microbiology, vol. 112, no. 2-4, pp. 163-169, 2006.

[6] T. Kuiken, J. van den Brand, D. van Riel, M. Pantin-Jackwood, and D. E. Swayne, "Comparative pathology of select agent influenza a virus infections," Veterinary Pathology, vol. 47, no. 5, pp. 893-914, 2010.

[7] S. Pillet, N. Svitek, and V. von Messling, "Ferrets as a model for morbillivirus pathogenesis, complications, and vaccines," Current Topics in Microbiology and Immunology, vol. 330, pp. 73-87, 2009.

[8] C. J. Sigurdson, C. K. Mathiason, M. R. Perrott et al., "Experimental chronic wasting disease (CWD) in the ferret," Journal of Comparative Pathology, vol. 138, no. 4, pp. 189-196, 2008.

[9] J. R. Ragg, H. Moller, and K. A. Waldrup, "The prevalence of bovine tuberculosis (Mycobacterium bovis) infections in feral populations of cats (Felis catus), ferrets (Mustela furo) and stoats (Mustela erminea) in Otago and Southland, New Zealand," New Zealand Veterinary Journal, vol. 43, no. 7, pp. 333-337, 1995.
[10] J. D. Rodgers, N. L. Connery, J. McNair et al., "Experimental exposure of cattle to a precise aerosolised challenge of Mycobacterium bovis: a novel model to study bovine tuberculosis," Tuberculosis, vol. 87, no. 5, pp. 405-414, 2007.

[11] R. A. Skuce, S. W. McDowell, T. R. Mallon et al., "Discrimination of isolates of Myobacterium bovis in Northern Ireland on the basis of variable numbers of tandem repeates (VNTRs)," Veterinary Record, vol. 157, no. 17, pp. 501-504, 2005.

[12] G. W. de Lisle, R. P. Kawakami, G. F. Yates, and D. M. Collins, "Isolation of Mycobacterium bovis and other mycobacterial species from ferrets and stoats," Veterinary Microbiology, vol. 132, no. 3-4, pp. 402-407, 2008.

[13] S. D. Neill, J. Cassidy, J. Hanna et al., "Detection of Mycobacterium bovis infection in skin test-negative cattle with an assay for bovine interferon-gamma," Veterinary Record, vol. 135, no. 6, pp. 134-135, 1994.

[14] D. Murphy, E. Gormley, E. Costello, D. O’Meara, and L. A. L. Corner, "The prevalence and distribution of Mycobacterium bovis infection in European badgers (Meles meles) as determined by enhanced post mortem examination and bacteriological culture," Research in Veterinary Science, vol. 88, no. 1, pp. 1-5, 2010.

[15] L. A. L. Corner, D. Murphy, and E. Gormley, "Mycobacterium bovis infection in the Eurasian badger (Meles meles): the disease, pathogenesis, epidemiology and control," Journal of Comparative Pathology, vol. 144, no. 1, pp. 1-24, 2011.

[16] L. A. Corner, "Personal communication," 2010.

[17] C. E. Stager, J. P. Libonati, S. H. Siddiqi et al., "Role of solid media when used in conjunction with the BACTEC system for mycobacterial isolation and identification," Journal of Clinical Microbiology, vol. 29, no. 1, pp. 154-157, 1991.

[18] L. A. Corner and C. Nicolacopoulos, "Comparison of media used for the primary isolation of Mycobacterium bovis by veterinary and medical diagnostic laboratories," Australian Veterinary Journal, vol. 65, no. 7, pp. 202-205, 1988.

[19] L. A. Corner, "Post mortem diagnosis of Mycobacterium bovis infection in cattle," Veterinary Microbiology, vol. 40, no. 1-2, pp. 53-63, 1994.

[20] C. S. Cox, The Aerobiological Pathway of Organisms, WileyInterscience Publications, Chichester, UK, 1987.

[21] D. W. Smith, D. N. McMurray, E. H. Wiegeshaus, A. A. Grover, and G. E. Harding, "Host-parasite relationships in experimental airborne tuberculosis. IV. Early events in the course of infection in vaccinated and nonvaccinated guinea pigs," American Review of Respiratory Disease, vol. 102, no. 6, pp. 937-949, 1970.

[22] I. W. Lugton, G. Wobeser, R. S. Morris, and P. Caley, "Epidemiology of Mycobacterium bovis infection in feral ferrets (Mustela furo) in New Zealand: II. Routes of infection and excretion," New Zealand Veterinary Journal, vol. 45, no. 4, pp. 151-157, 1997.

[23] J. Gallagher, R. Monies, M. Gavier-Widen, and B. Rule, "Role of infected, non-diseased badgers in the pathogenesis of tuberculosis in the badger," Veterinary Record, vol. 142, no. 26, pp. 710-714, 1998.

[24] J. Gallagher and P. A. Jenkins, "Mycobacterial diseases," in Zoonoses, pp. 155-164, Oxford University Press, Oxford, UK, 1998.

[25] R. S. Clifton-Hadley, "Badgers, bovine tuberculosis and the age of reason," The British Veterinary Journal, vol. 152, no. 3, pp. 243-246, 1996. 
[26] L. A. Corner, A. C. Trajstman, and K. Lund, "Determination of the optimum concentration of decontaminants for the primary isolation of Mycobacterium bovis," New Zealand Veterinary Journal, vol. 43, pp. 129-133, 1995.

[27] J. R. Ragg, K. A. Waldrup, and H. Moller, "The distribution of gross lesions of tuberculosis caused by Mycobacterium bovis in feral ferrets (Mustela furo) from Otago, New Zealand," New Zealand Veterinary Journal, vol. 43, no. 7, pp. 338-341, 1995.

[28] I. W. Lugton, G. Wobeser, R. S. Morris, and P. Caley, "Epidemiology of Mycobacterium bovis infection in feral ferrets (Mustela furo) in New Zealand: I. Pathology and diagnosis," New Zealand Veterinary Journal, vol. 45, no. 4, pp. 140-150, 1997.

[29] J. M. Pollock, J. D. Rodgers, M. D. Welsh, and J. McNair, "Pathogenesis of bovine tuberculosis: the role of experimental models of infection," Veterinary Microbiology, vol. 112, no. 2-4, pp. 141-150, 2006.

[30] M. L. Cross, R. E. Labes, and C. G. Mackintosh, "Oral infection of ferrets with virulent Mycobacterium bovis or Mycobacterium avium: susceptibility, pathogenesis and immune response," Journal of Comparative Pathology, vol. 123, no. 1, pp. 15-21, 2000.

[31] T. Qureshi, R. E. Labes, M. L. Cross, J. F. T. Griffin, and C. G. Mackintosh, "Partial protection against oral challenge with Mycobacterium bovis in ferrets (Mustela furo) following oral vaccination with BCG," International Journal of Tuberculosis and Lung Disease, vol. 3, no. 11, pp. 1025-1033, 1999. 

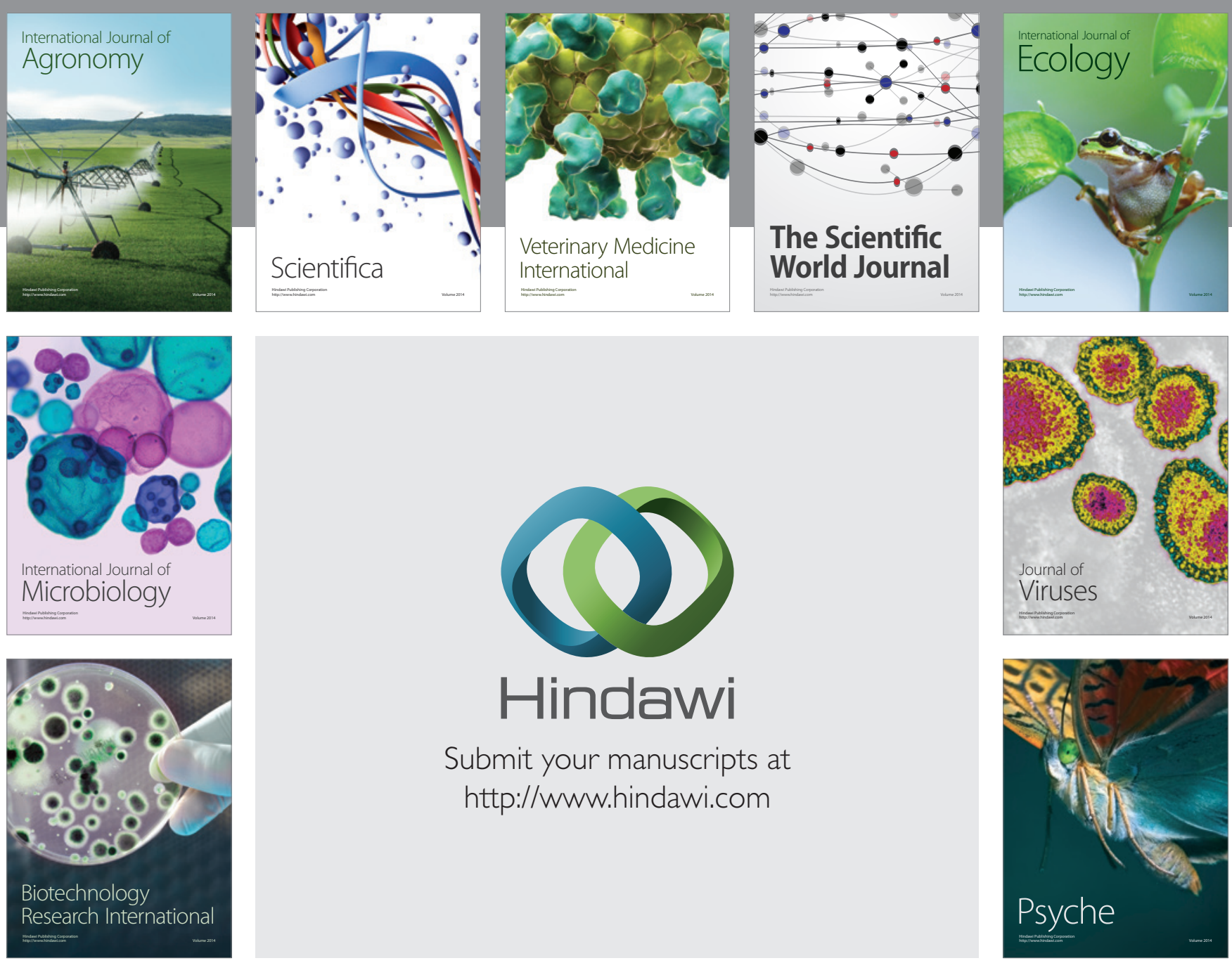

Submit your manuscripts at

http://www.hindawi.com
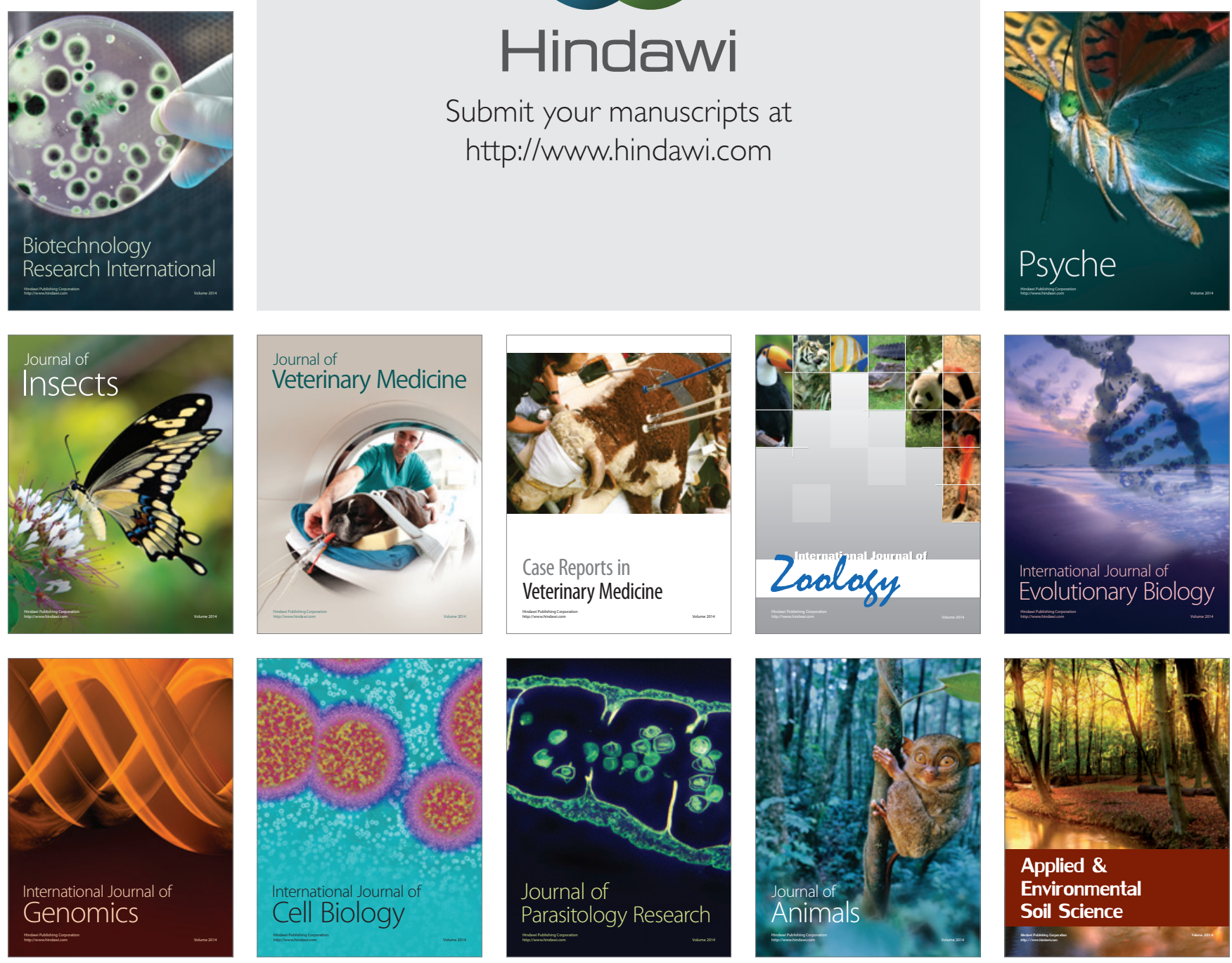